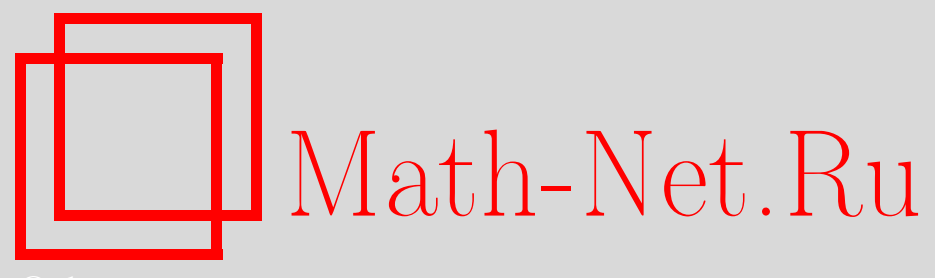

В. Брагин, Одноцветный треугольник площади 1 на покрашенной плоскости, Квант, 2021, номер 5, 37-38

DOI: https://doi.org/10.4213/kvant20210503

Использование Общероссийского математического портала Math-Net.Ru подразумевает, что вы прочитали и согласны с пользовательским соглашением http://www.mathnet.ru/rus/agreement

Параметры загрузки:

IP : 35.173 .137 .237

26 апреля 2023 г., 14:04:20 


\section{Одноцветный треугольник площади 1 на покрашенной плоскости}

\section{В.Брагин}

Эта статья посвящена решению следующей трудной задачи с простым условием (см. «Квант» № 8 за 2020 г.):

М2617. Плоскость покрашена в 100 цветов. Докажите, что найдутся три точки одного ивета, являющиеся вершинами треугольника площади 1.

В решении этой задачи мне посчастливилось использовать классическую теорему Ван дер Вардена:

Для любых натуральных $k$ u $r$ существуует натуральное число $W(k ; r)$ со следующим свойством: если раскрасить элементы арифметической прогрессии длины $W(k ; r)$ произвольным образом в $k$ цветов, то среди них найдется одноцветная арифметическая прогрессия длины $r$.

Далее арифметической прогрессией прямых длины $n$ назовем последовательность различных параллельных прямых $l_{1}, l_{2}, \ldots, l_{n}$ такую, что расстояния между парами прямых с соседними номерами равны (рис. 1).

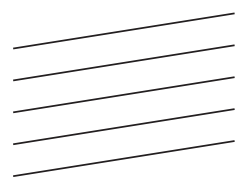

Puc. 1
Теперь будем раскрашивать не числа, а точки. Докажем утверждение, из которого задача очевидно следует.

Теорема. Для любого натурального $n$ существует натуральное L $(n)$, обладаюшие следуюшим свойством. Пусть дана арифметическая прогрессмя из $L(n)$ nрямых, и пусть каждая точка на кажддой из этих прямых окрашена в один из $n$ чветов. Тогда найдутся три точки одного ивета, лежащие на прямых из этой прогрессии, являюшиеся вершинами треугольника площади 1.

Доказательство будем вести по индукции.

База: $n=1$. Достаточно взять $L(1)=2$. Если есть две прямые, полностью покрашенные в один цвет, то очевидно найдется одноцветный треугольник любой площади.

DOI: https://doi.org/10.4213/kvant20210503
Сделаем переход от $n$ к $n+1$. Окажется, что можно положить

$$
L(n+1)=(2 L(n) !) ! \cdot W(n+1 ; 2 L(n) !) !+1
$$

(да!! тут очень много факториалов).

Рассмотрим арифметическую прогрессию из $L(n+1)$ прямых, где расстояние между соседними прямыми равно $h$, такую, что на этих прямых есть только $n+1$ цвет. Предположим, что одноцветного треугольника площади 1 с вершинами на этих прямых нет, и докажем, что тогда найдется арифметическая прогрессия из $L(n)$ прямых, на которых одного из цветов совсем нет, после этого воспользуемся предположением индукции.

Обозначим $N=2 L(n) !, K=W(n+1 ; N)$, $M=N ! \cdot K !$, так что $L(n+1)=M+1$.

Рассмотрим арифметическую прогрессию из $K$ точек $x_{1}, \ldots, x_{K}$ на прямой $l_{1}$ (рис. 2). Разность $t$ этой прогрессии выберем равной $\frac{1}{2 h M}$. По теореме Ван дер Вардена, из этих $K$ точек,

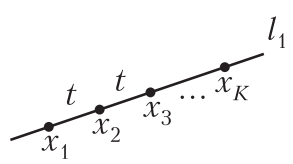

PUC. 2 покрашенных в $n+1$ цвет, можно выбрать одноцветную арифметическую прогрессию из $N$ точек, для определенности красного цвета. Разность этой прогрессии равна $S t$, где $S$ - натуральное число, не превышающее $K$. Тогда прямые, параллельные $l_{1}$, находящиеся от нее на расстояниях $\frac{1}{2 S t}, \frac{1}{2 \cdot 2 S t}, \ldots$ $\ldots, \frac{1}{(N-1) \cdot 2 S t}$, совсем не содержат точек красного цвета, иначе получился бы треугольник площади 1 с вершинами красного цвета. Кроме того, все эти прямые содержатся среди наших, потому что расстояния от всех упомянутых прямых равны $\frac{1}{2 a S t}$, где $1 \leq a \leq N-1$, а частные этих чисел при делении на $h$ равны

$$
\frac{1}{2 a S t h}=\frac{2 h M}{2 a S h}=\frac{M}{a S}=\frac{N !}{a} \cdot \frac{K !}{S} .
$$

Из выражения $\frac{N !}{a} \cdot \frac{K !}{S}$ видно, что эти частные целые, а из $\frac{M}{a S}-$ что они меньше $M$. 
Значение $N$ подобрано с таким расчетом, чтобы из нашего множества прямых, не содержащих красных точек, нашлось $L(n)$ прямых, образующих арифметическую прогрессию. Действительно, достаточно среди них выбрать прямые, находящиеся от $l_{1}$ на расстояниях $\frac{1}{N \cdot S t}, \frac{1}{(N / 2) \cdot S t}$, 1 $\overline{(N / 3) \cdot S t}, \ldots, \overline{(N / L(n)) \cdot S t}$.

Этим завершается доказательство теоремы, и задача М2617 решена.

Расскажем теперь о том, как это решение придумывалось. Зачастую очень важно не только читать строгое, четкое и аккуратное решение задачи, но и осознать ключевые идеи, показанные «на пальцах».

К решению задачи я подошел с другой стороны. Если я найду на какой-то прямой $l$ одноцветную арифметическую прогрессию длины $k$ с разностью $2 d$, то найдутся $k-1$ прямых, параллельных $l$ и лежащих по одну сторону от $l$ на расстояниях $\frac{1}{2 d}$, $\frac{1}{4 d}, \frac{1}{6 d}, \ldots, \frac{1}{2(k-1) d}$ от $l$, на которых этого цвета не будет. Читатель заметит, что это не арифметическая прогрессия прямых. Собственно, у меня на этом этапе мыслительного процесса еще и не было понимания, что именно ее надо искать. Но потом это понимание появилось, когда я понял, что надо понемногу исключать цвета, оставляя достаточно много параллельных прямых, на которых этих цветов нет. Но если это будут прямые без всякой структуры, то неясно, как потом исключать цвета. После этого родилась идея формулировки теоремы, которая позволила «обуздать хаос». Далее я понял, что $k$ должно быть столь велико, чтобы среди чисел $\frac{1}{2 d}, \frac{1}{4 d}$, $\frac{1}{6 d}, \ldots, \frac{1}{2(k-1) d}$ нашлась арифметическая прогрессия длины $L(n)$. Как такое $k$ подобрать, я уже знал из «олимпиадного фольклора». Например, можно взять $k-1>L(n)$. Тогда числа $\frac{1}{L(n) !}, \frac{2}{L(n) !}$, $\frac{3}{L(n) !}, \ldots, \frac{1}{(L(n)-1) !}$ образуют арифмети- ческую прогрессию длины $L(n)$ с разностью $\frac{1}{L(n) !}$. Следующая проблема в том, что я заранее не знаю, какое $d$ получится. К счастью, теорема Ван дер Вардена говорит, что таких $d$ конечное количество, поэтому в моей прогрессии с $n+1$ цветом должны быть все прямые на расстояниях $\frac{1}{2 d}, \frac{1}{4 d}, \frac{1}{6 d}, \ldots, \frac{1}{2(k-1) d}$ для всех возможных $d$. Поскольку все возможные значения $d$ все же соизмеримы (т.е. отношение любых двух рационально), а если точнее, $2 d$ принимает значения от 1 до $W(n+1 ; 2 L(n) !)($ с запасом $)$, то существует некоторое рациональное число, которое укладывается целое количество раз в каждом из встретившихся нам расстояний. Например, числа 1/100, 1/1001, 1/17 «делятся» на $1 /(100 \cdot 1001 \cdot 17)$, т.е. достаточно взять дробь 1/(произведение всех знаменателей). Это соображение и выводит на финишную прямую в конструировании $L(n+1)$. Это число должно делиться на все знаменатели, которые встретятся. Поэтому можно взять $L(n+1)=$ $=(2 L(n) !) ! \cdot(W(n+1 ; 2 L(n) !)) !$ Действительно, второй множитель точно делится на любое $d$, а первый точно делится на то, на что $d$ домножается в знаменателе в дробях $\frac{1}{2 d}, \frac{1}{4 d}, \frac{1}{6 d}, \ldots, \frac{1}{2(k-1) d}$.

Читатель, не столь привыкший читать тексты с громоздкими выкладками, мог быть озадачен, увидев большие формулы в самом начале. Но появились эти константы далеко не сразу, а когда задача уже была решена и почти записана. При чтении многих решений задач полезно понимать, что придуманы они были в совсем другом виде. А иногда можно по решению задачи попробовать восстановить, как оно могло быть придумано. 